Kyushu J. Math.

Vol. 58, 2004, pp. 59-70

\title{
MAXIMAL SURFACES WITH SIMPLE ENDS
}

\author{
Taishi IMAIZUMI
}

(Received 17 October 2002 and revised 28 December 2002 and 24 April 2003)

\section{Introduction}

A maximal surface in the three-dimensional Minkowski space $\mathbf{L}^{3}$ is a space-like surface with zero mean curvature. It is well-known that there is a Weierstrass-type representation for maximal surfaces, that is, a representation formula in terms of holomorphic data similar to the Weierstrass representation for minimal surfaces in the Euclidean 3-space $\mathbf{E}^{3}$ (Lemma 1.3). Using the holomorphic data $(g, \omega)$ of the Weierstrass-type representation for a maximal surface, we see that the induced metric degenerates on the set $\{|g|=1\}$. On the other hand, it is well-known that there exist no complete maximal surfaces in $\mathbf{L}^{3}$ except space-like planes. So we consider a class of surfaces which admit degenerate points of the induced metric (Definition 2.1).

For a minimal surface in $\mathbf{E}^{3}$, the following fact is well-known: Let $f: \Delta^{*} \rightarrow \mathbf{E}^{3}$ be a conformal minimal immersion of a punctured disc $\Delta^{*}=\{z \in \mathbb{C} ; 0<|z|<1\}$ into $\mathbf{E}^{3}$ which is complete at the origin 0 . Then ord $\operatorname{or}_{z=0} d s^{2}=-2$ holds if and only if the end 0 is asymptotic to a catenoid or a plane in $\mathbf{E}^{3}$ [6]. Analogous to this fact, we introduce the simple end for maximal maps (Definition 2.2).

In this paper, we consider a maximal map with simple ends and show the following theorem (Theorem 2.7).

Theorem. Let $\Delta:=\{z \in \mathbb{C} ;|z|<1\}$ and $\Delta^{*}:=\Delta \backslash\{0\}$. Assume $f: \Delta^{*} \rightarrow \mathbf{L}^{3}$ is a maximal map such that the end 0 is simple. Then $f$ is asymptotic to one of the following three types:

(I) $\left(a r^{-1} \cos \theta, a r^{-1} \sin \theta, c \log r\right)$

(II) $\left(a r^{-1} \sin \theta, c \log r, a r^{-1} \sin \theta\right)$,

(III) $\left(b r^{-1} \cos \theta+c \log r, 0,-b r^{-1} \cos \theta-c \log r\right)$,

on a neighborhood of 0 , where $z:=r e^{\sqrt{-1} \theta}, a, b \in \mathbb{R} \backslash\{0\}$, and $c \in \mathbb{R}$.

In the final section, we consider the flux vector of maximal maps similarly to the case of minimal surfaces in $\mathbf{E}^{3}$ (Definition 3.1), and we show the relationship between the simple ends and their flux (Theorem 3.3). 


\section{Preliminaries}

Let $\mathbf{L}^{3}$ denote the three-dimensional Minkowski space, that is, the real vector space $\mathbb{R}^{3}$ endowed with the Lorentz metric

$$
\langle\cdot, \cdot\rangle=\left(d x^{1}\right)^{2}+\left(d x^{2}\right)^{2}-\left(d x^{3}\right)^{2},
$$

where $\left(x^{1}, x^{2}, x^{3}\right)$ are the canonical coordinates in $\mathbb{R}^{3}$. An immersion $f: M \rightarrow \mathbf{L}^{3}$ of a two-dimensional manifold $M$ is said to be space-like if the pull-back $d s^{2}$ of $\langle\cdot, \cdot\rangle$ by $f$ is a Riemannian metric on $M$. Let $v$ be the unit normal vector field of a space-like immersion $f$, namely at each point $p \in M, v_{p}$ is perpendicular to the tangent plane and $\left\langle v_{p}, v_{p}\right\rangle=-1$. The second fundamental form is a quadratic form on $M$ defined by

$$
h=\langle d f, d v\rangle .
$$

Then the mean curvature is defined as a half the trace of $h$ with respect to $d s^{2}$.

Definition 1.1. A space-like immersion $f$ is said to be maximal if the mean curvature of $f$ vanishes identically.

A space-like immersion $f: M \rightarrow \mathbf{L}^{3}$ induces a conformal structure on $M$ by $d s^{2}$. Hence, if $M$ is orientable, $f$ can be regarded as a conformal immersion of a Riemann surface $M$.

Let $H^{2}$ be a space-like surface in $\mathbf{L}^{3}$ defined by

$$
\begin{aligned}
H^{2} & =\left\{\left(x^{1}, x^{2}, x^{3}\right) \in \mathbf{L}^{3} ;\left(x^{1}\right)^{2}+\left(x^{2}\right)^{2}-\left(x^{3}\right)^{2}=-1\right\} \\
& =\left\{\left(x^{1}, x^{2}, x^{3}\right) \in H^{2} ; x^{3}>0\right\} \cup\left\{\left(x^{1}, x^{2}, x^{3}\right) \in H^{2} ; x^{3}<0\right\} \\
& =H_{+}^{2} \cup H_{-}^{2} .
\end{aligned}
$$

If $f: M \rightarrow \mathbf{L}^{3}$ is a space-like immersion, the unit normal vector $v$ of $f$ determines a point of $H^{2}$ at each point of $M$.

The map

$$
\sigma: H^{2} \rightarrow \mathbb{C} \cup\{\infty\} \backslash\{z \in \mathbb{C} ;|z|=1\}
$$

defined by

$$
\sigma\left(x^{1}, x^{2}, x^{3}\right)=\frac{1}{1-x^{3}}\left(x^{1}+\sqrt{-1} x^{2}\right)
$$

is conformal. The map $\sigma$ is called the stereographic projection.

Definition 1.2. For a space-like immersion $f: M \rightarrow \mathbf{L}^{3}$, the map

$$
g: M \rightarrow \mathbb{C} \cup\{\infty\} \backslash\{z \in \mathbb{C} ;|z|=1\} ; \quad g(p)=\sigma\left(v_{p}\right)
$$


is called the Gauss map of $f$, where $v_{p}$ is the unit normal vector of $f$ at $p$, and $\sigma$ is the stereographic projection as in (1.1).

It is well-known that a maximal immersion can be represented in terms of holomorphic data.

LEMmA 1.3. (Weierstrass-type representation [4]) Let $M$ be a Riemann surface and $f: M \rightarrow \mathbf{L}^{3}$ a conformal maximal immersion. Then there exist a meromorphic function $g: M \rightarrow \mathbb{C} \cup\{\infty\} \backslash\{\zeta \in \mathbb{C} ;|\zeta|=1\}$ and a holomorphic 1-form $\omega$ on $M$ such that

$$
f(z)=\operatorname{Re} \int_{z_{0}}^{z} \alpha, \quad \alpha=\left(1+g^{2}, \sqrt{-1}\left(1-g^{2}\right),-2 g\right) \omega,
$$

where $z$ is a complex coordinate on $M$ and $z_{0} \in M$ is a base point.

Moreover, $g$ is the Gauss map of $f$, and the induced metric of $f$ is written as

$$
d s^{2}=\left(1-|g|^{2}\right)^{2}|\omega|^{2} .
$$

Conversely, let $g: M \rightarrow \mathbb{C} \cup\{\infty\} \backslash\{z \in \mathbb{C} ;|z|=1\}$ be a meromorphic function and $\omega$ a holomorphic 1-form on $M$, such that $d s^{2}$ in (1.3) is non-degenerate.

If

$$
\operatorname{Re} \oint_{\gamma} \alpha=0
$$

for any loops $\gamma$ on $M$, then there exists a maximal immersion $f: M \rightarrow \mathbf{L}^{3}$ such that $f$ is given by (1.2).

We call the pair $(g, \omega)$ in Lemma 1.3 the Weierstrass data of $f$.

\section{Maximal map}

If $g: M \rightarrow \mathbb{C} \cup\{\infty\}$ is a meromorphic function, then the map $f$ given by (1.2) degenerates on

$$
\{z \in M ;|g(z)|=1\} .
$$

So we consider a notion of maximal surfaces as a surface with degenerate points.

Definition 2.1. Let $M$ be a Riemann surface and $g$ (respectively $\omega$ ) a meromorphic function (respectively meromorphic 1-form) on $M$. We denote by $P$ the set of poles of

$$
\alpha=\left(1+g^{2}, \sqrt{-1}\left(1-g^{2}\right),-2 g\right) \omega
$$


and $S:=\{z \in M ;|g(z)|=1\}$. Then a map $f: M^{*}:=M \backslash P \rightarrow \mathbf{L}^{3}$ given by (1.2) is called a maximal map with Weierstrass data $(g, \omega)$ if:

(1) $P$ is a discrete set of $M$;

(2) the restriction $\left.f\right|_{M^{*} \backslash S}$ is a conformal maximal immersion;

(3) the Weierstrass data of $\left.f\right|_{M^{*} \backslash S}$ is $(g, \omega)$.

We call a point $q \in P$ an $e n d$ of $f$.

For a minimal immersion $f$ in $\mathbf{E}^{3}$ with Weierstrass data $(g, \omega)$, if $g$ is one-to-one on a neighborhood of an end of $f$ and $\omega d g$ has a pole of order two at the end of $f$, then $f$ is asymptotic to a catenoid on the neighborhood of the end of $f$. So, we introduce the following notion for ends of maximal maps.

Definition 2.2. An end $q \in P$ of maximal map with Weierstrass data $(g, \omega)$ is called simple if and only if the $\mathbb{C}^{3}$-valued 1 -form $\alpha$ in (2.1) has a pole of order two at $q$.

Example 2.3. [5] Let $M^{*}:=\mathbb{C} \cup\{\infty\} \backslash\{0, \infty\}$ and $(g, \omega)=\left(z, a z^{-2} d z\right)$, where $a \in \mathbb{R} \backslash\{0\}$. Then $(g, \omega)$ induces a maximal map $f_{1}$ :

$$
\begin{aligned}
f_{1}(z) & =\operatorname{Re} \int\left(a\left(\frac{1}{z^{2}}+1\right), \sqrt{-1} a\left(\frac{1}{z^{2}}-1\right),-\frac{2 a}{z}\right) d z \\
& =a \operatorname{Re}\left(-\frac{1}{z}+z,-\sqrt{-1}\left(\frac{1}{z}+z\right),-2 \log z\right) .
\end{aligned}
$$

Let $z=r e^{\sqrt{-1} \theta}(r>0,-\pi \leq \theta \leq \pi)$. Then

$$
f_{1}=a\left(\left(r-\frac{1}{r}\right) \cos \theta,\left(r-\frac{1}{r}\right) \sin \theta,-2 \log r\right) .
$$

Therefore, $f_{1}$ is asymptotic to

$$
a\left(-\frac{1}{r} \cos \theta,-\frac{1}{r} \sin \theta,-2 \log r\right)
$$

on a neighborhood of $z=0$.

The image of $f_{1}$ is

$$
f_{1}\left(M^{*}\right)=\left\{\left(x^{1}, x^{2}, x^{3}\right) \in \mathbf{L}^{3} ;\left(x^{1}\right)^{2}+\left(x^{2}\right)^{2}-4 a^{2} \sinh ^{2}\left(\frac{x^{3}}{2 a}\right)=0\right\} .
$$

(See Figure 1.) 


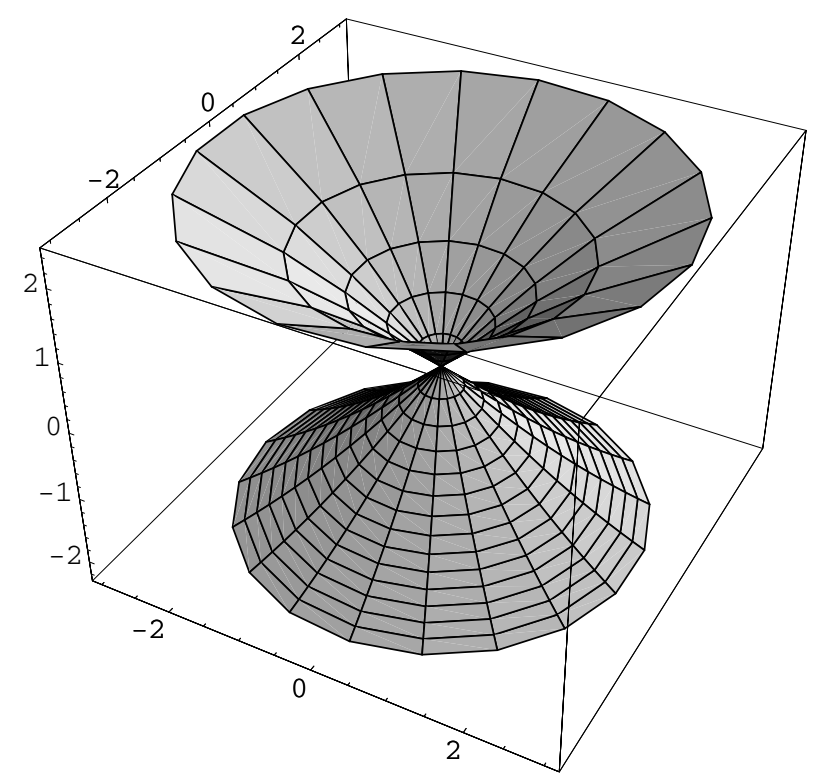

FIGURE 1. The surface in Example 2.3 (called the Lorentz catenoid).

Example 2.4. [5] Let $M^{*}:=\mathbb{C} \cup\{\infty\} \backslash\{1,-1\}$ and

$$
(g, \omega)=\left(z, \frac{\sqrt{-1} b}{(z-1)^{2}(z+1)^{2}} d z\right)
$$

where $b \in \mathbb{R} \backslash\{0\}$.

Then $(g, \omega)$ induces a maximal map $f_{2}$ :

$$
f_{2}(z)=b \operatorname{Re}\left(-\sqrt{-1} \frac{z}{(z+1)(z-1)},-\frac{1}{2} \log \frac{z+1}{z-1}, \sqrt{-1} \frac{1}{(z+1)(z-1)}\right)
$$

Let $w=(z+1)(z-1)^{-1}$. Then

$$
f_{2}(w)=b \operatorname{Re}\left(-\frac{\sqrt{-1}}{4} \frac{w^{2}-1}{w},-\frac{1}{2} \log w, \frac{\sqrt{-1}}{4} \frac{(w-1)^{2}}{w}\right) .
$$

Moreover, let $w=r e^{\sqrt{-1} \theta}(r>0,-\pi \leq \theta \leq \pi)$. Then

$$
f_{2}(r, \theta)=b\left(\frac{1}{4}\left(r+\frac{1}{r}\right) \sin \theta,-\frac{1}{2} \log r,-\frac{1}{4}\left(r-\frac{1}{r}\right) \sin \theta\right) .
$$




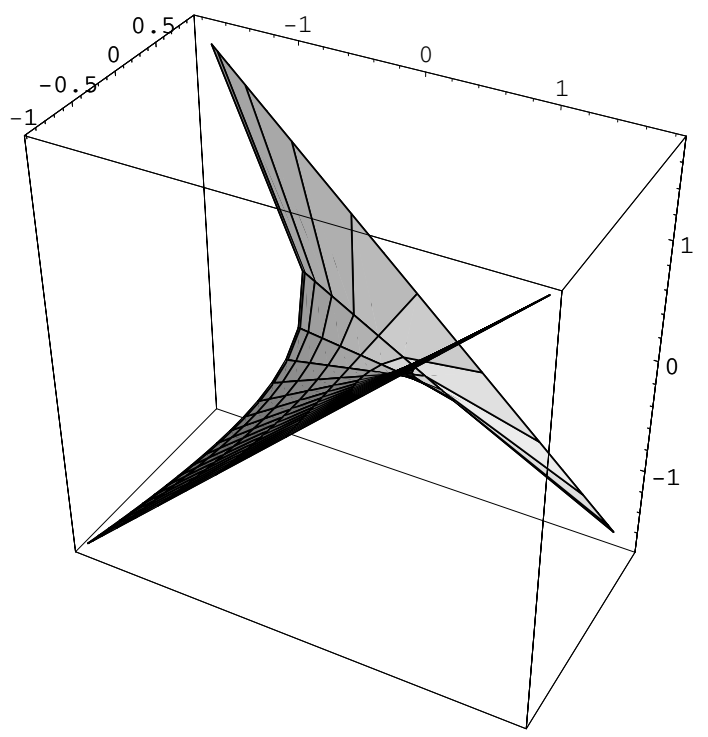

FIGURE 2. The surface in Example 2.4.

Therefore, $f_{2}$ is asymptotic to

on a neighborhood of $z=-1$.

$$
\frac{b}{4}\left(\frac{1}{r} \sin \theta,-2 \log r, \frac{1}{r} \sin \theta\right)
$$

The image of $f_{2}$ is

$$
f_{2}\left(M^{*}\right)=\left\{\left(x^{1}, x^{2}, x^{3}\right) \in \mathbf{L}^{3} ; x^{3}-x^{1} \tanh \left(\frac{2 x^{2}}{b}\right)=0,\left|x^{1}\right| \leq \frac{b}{2} \cosh \frac{2 x^{2}}{b}\right\} .
$$

(See Figure 2.)

Example 2.5. Let $M^{*}:=\mathbb{C} \cup\{\infty\} \backslash\{0,1,-1\}$ and

$$
(g, \omega)=\left(\frac{2 z}{z^{2}+1}, \frac{c\left(z^{2}+1\right)^{2}}{z^{2}\left(z^{2}-1\right)^{2}} d z\right)
$$

where $c \in \mathbb{R} \backslash\{0\}$. Then $(g, \omega)$ induces a maximal map $f_{3}$ :

$$
f_{3}(z)=-c \operatorname{Re}\left(\frac{5 z^{2}-1}{z\left(z^{2}-1\right)}+2 \log \frac{z-1}{z+1}, \frac{\sqrt{-1}}{z},-\frac{4}{z^{2}-1}+2 \log \frac{z^{2}}{z^{2}-1}\right) .
$$




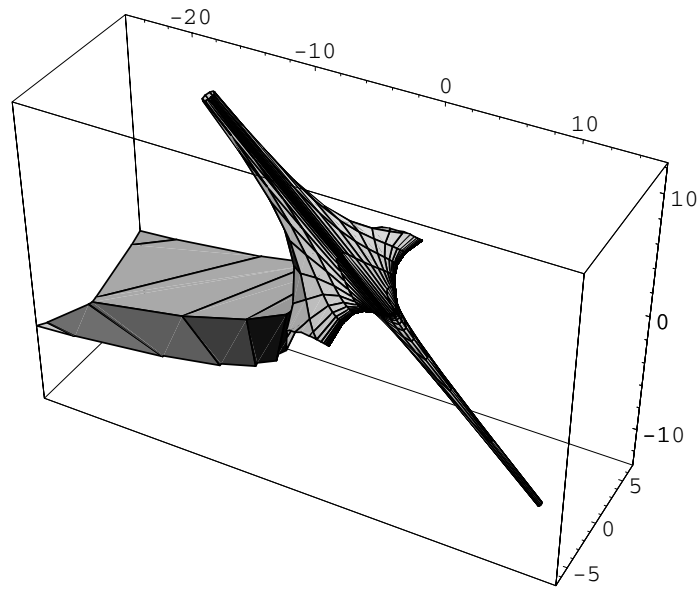

FIgURE 3. The surface in Example 2.5.

Let $z-1=r e^{\sqrt{-1} \theta}(r>0,-\pi \leq \theta \leq \pi)$. Then it is seen that $f_{3}$ is asymptotic to

$$
-2 c\left(\frac{1}{r} \cos \theta+\log r, 0,-\frac{1}{r} \cos \theta-\log r\right)
$$

on a neighborhood of $z=1$. (See Figure 3.)

Remark 2.6. Even if the order of $\alpha$ at an end is -2 , the order of $\omega d g$ at the end is not always equal to -2 . (See Example 2.5.)

In the following theorem, we prove that any simple end is asymptotic to one of the ends shown in Examples 2.3, 2.4 and 2.5 or a space-like plane or a null line.

Theorem 2.7. Let $\Delta:=\{z \in \mathbb{C} ;|z|<1\}$ and $\Delta^{*}:=\Delta \backslash\{0\}$. Assume $f: \Delta^{*} \rightarrow \mathbf{L}^{3}$ is a maximal map such that the end 0 is simple. Then $f$ is asymptotic to one of the following three types:

(I) $\left(a r^{-1} \cos \theta, a r^{-1} \sin \theta, c \log r\right)$,

(II) $\left(a r^{-1} \sin \theta, c \log r, a r^{-1} \sin \theta\right)$,

(III) $\left(b r^{-1} \cos \theta+c \log r, 0,-b r^{-1} \cos \theta-c \log r\right)$,

on a neighborhood of 0 , where $z:=r e^{\sqrt{-1} \theta}, a, b \in \mathbb{R} \backslash\{0\}$, and $c \in \mathbb{R}$.

Proof. By the definition of a maximal map, $f$ can be written as

$$
f=\operatorname{Re} \int \alpha
$$


where $\alpha$ is as in (2.1). Since 0 is a simple end, the order of $\alpha$ at 0 is -2 . Hence $\alpha$ can be expanded as the following:

$$
\alpha=\left(\frac{1}{z^{2}} \boldsymbol{a}_{-2}+\frac{1}{z} \boldsymbol{a}_{-1}+\cdots\right) d z
$$

where $\boldsymbol{a}_{-2}, \boldsymbol{a}_{-1}, \ldots \in \mathbb{C}^{3}$, and $\boldsymbol{a}_{-2} \neq \mathbf{0}$.

By (2.1), $\langle\alpha, \alpha\rangle_{\mathbb{C}}=0$, where $\langle\cdot, \cdot\rangle_{\mathbb{C}}$ is the complexification of the Lorentz metric $\langle\cdot, \cdot\rangle$. Then we have

$$
\frac{1}{z^{4}}\left\langle\boldsymbol{a}_{-2}, \boldsymbol{a}_{-2}\right\rangle_{\mathbb{C}}+\frac{2}{z^{3}}\left\langle\boldsymbol{a}_{-2}, \boldsymbol{a}_{-1}\right\rangle_{\mathbb{C}}+\cdots=0
$$

that is,

$$
\left\{\begin{array}{l}
\left\langle\boldsymbol{a}_{-2}, \boldsymbol{a}_{-2}\right\rangle_{\mathbb{C}}=0, \\
\left\langle\boldsymbol{a}_{-2}, \boldsymbol{a}_{-1}\right\rangle_{\mathbb{C}}=0 .
\end{array}\right.
$$

Since

$$
\operatorname{Re} \oint_{\gamma} \alpha=0
$$

holds for any loop $\gamma$ surrounding $0, \boldsymbol{a}_{-1}$ is real vector.

Therefore, if we set $\boldsymbol{a}+\sqrt{-1} \boldsymbol{b}:=\boldsymbol{a}_{-2}, \boldsymbol{c}:=\boldsymbol{a}_{-1},(\boldsymbol{a}, \boldsymbol{b}, \boldsymbol{c}$ are real vectors $)$ such that $(\boldsymbol{a}, \boldsymbol{b}) \neq(\mathbf{0}, \mathbf{0})$, then (2.2) is equivalent to the following conditions:

$$
\left\{\begin{array}{l}
\langle\boldsymbol{a}, \boldsymbol{a}\rangle=\langle\boldsymbol{b}, \boldsymbol{b}\rangle, \\
\langle\boldsymbol{a}, \boldsymbol{b}\rangle=0, \\
\langle\boldsymbol{a}, \boldsymbol{c}\rangle=\langle\boldsymbol{b}, \boldsymbol{c}\rangle=0 .
\end{array}\right.
$$

Then the triple $(\boldsymbol{a}, \boldsymbol{b}, \boldsymbol{c})$ is one of the cases in Table 1.

In the case of (I), we may set, without loss of generality, that

$$
\boldsymbol{a}=-a(1,0,0), \quad \boldsymbol{b}=-a(0,1,0), \quad \boldsymbol{c}=c(0,0,1),
$$

where $a, c \in \mathbb{R}, a \neq 0$. Hence

$$
\begin{gathered}
\alpha=\left(-\frac{a}{z^{2}}(1, \sqrt{-1}, 0)+\frac{c}{z}(0,0,1)+\cdots\right) d z \\
\int \alpha=\frac{a}{z}(1, \sqrt{-1}, 0)+c \log z(0,0,1)+o(1) . \\
\text { Set } z=r e^{\sqrt{-1} \theta}(r>0,-\pi \leq \theta \leq \pi), \text { then } \\
f=\operatorname{Re} \int \alpha=\left(a r^{-1} \cos \theta, a r^{-1} \sin \theta, c \log r\right)+o(1) .
\end{gathered}
$$

Similarly, in the cases of (II-i)-(III-iii), $f$ is asymptotic to the following: 
TABLE 1. Types of triple $(\boldsymbol{a}, \boldsymbol{b}, \boldsymbol{c})$.

\begin{tabular}{cccc}
\hline Type & $\boldsymbol{c}$ & $\boldsymbol{a}$ & $\boldsymbol{b}$ \\
\hline (I) & Time-like or 0 & Space-like & Space-like \\
(II-i) & Space-like or 0 & $\mathbf{0}$ & Null \\
(II-ii) & Space-like or 0 & Null & $\mathbf{0}$ \\
(II-iii) & Space-like or 0 & Null & Null \\
(III-i) & Null or 0 & $\mathbf{0}$ & Null \\
(III-ii) & Null or 0 & Null & $\mathbf{0}$ \\
(III-iii) & Null or 0 & Null & Null \\
\hline
\end{tabular}

(II-i) $\quad\left(a r^{-1} \sin \theta, c \log r, a r^{-1} \sin \theta\right)$;

(II-ii) $\quad\left(a r^{-1} \cos \theta, c \log r, a r^{-1} \cos \theta\right)$;

(II-iii) $\quad\left(r^{-1}(a \cos \theta+b \sin \theta), c \log r, r^{-1}(a \cos \theta+b \sin \theta)\right)$;

(III-i) $\quad\left(b r^{-1} \sin \theta+c \log r, 0,-b r^{-1} \sin \theta-c \log r\right)$;

(III-ii) $\quad\left(b r^{-1} \cos \theta+c \log r, 0,-b r^{-1} \cos \theta-c \log r\right)$;

(III-iii) $\left(r^{-1}(a \cos \theta+b \sin \theta)+c \log r, 0,-r^{-1}(a \cos \theta+b \sin \theta)-c \log r\right)$.

By the addition formula of the trigonometric function, we have (II-i) $=($ II-ii) $=$ $($ II-iii $),($ III-i $)=($ III-ii $)=($ III-iii $)$.

Remark 2.8. In (I) in Theorem 2.7, if $c=0$, then $f$ is asymptotic to a space-like plane. Similarly, in the cases of (II) and (III), $f$ is asymptotic to a null line.

Remark 2.9. For a minimal immersion $f: M \rightarrow \mathbf{E}^{3}$, it is well-known that the Weierstrass representation is

$$
f(z)=\operatorname{Re} \int_{z_{0}}^{z} \tilde{\alpha}, \quad \tilde{\alpha}=\left(1-g^{2}, \sqrt{-1}\left(1+g^{2}\right), 2 g\right) \omega,
$$

and that, if $f$ is a complete minimal immersion with embedded ends, then the order of $\tilde{\alpha}$ at the end is equal to -2 . However, in our case, we can consider the case that the order of $\alpha$ at the end is equal to -1 . 
If the order $\alpha$ at the end 0 is -1 , then

$$
\alpha=\left(\frac{1}{z} \boldsymbol{a}_{-1}+\cdots\right) d z
$$

where $\boldsymbol{a}_{-1}$ is non-zero real vector.

Since $\langle\alpha, \alpha\rangle_{\mathbb{C}}=0,\left\langle\boldsymbol{a}_{-1}, \boldsymbol{a}_{-1}\right\rangle=0$. That is, $\boldsymbol{a}_{-1}$ is the null vector. We may set, without loss of generality, that $\boldsymbol{a}_{-1}=a(1,0,1), a \in \mathbb{R} \backslash\{0\}$. Hence

$$
\begin{aligned}
& \alpha=\left(\frac{1}{z}(1,0,1)+\cdots\right) d z, \\
& \int \alpha=a(1,0,1) \log z+o(1) .
\end{aligned}
$$

Therefore,

$$
f=\operatorname{Re} \int \alpha=(a \log r, 0, a \log r)+o(1),
$$

where $z=r e^{\sqrt{-1} \theta}(r>0,-\pi \leq \theta \leq \pi)$.

For example, $M^{*}:=\mathbb{C} \cup\{\infty\} \backslash\{0, \infty\}$ and $(g, \omega)=\left(z+1, z^{-1} d z\right)$.

\section{Flux}

In this section, we consider the relationship between the flux and the simple ends.

Definition 3.1. Let $M,(g, \omega)$ and $P$ be as in Definition 2.1. Then, for a simple end $q \in P$ of a maximal map $f: M^{*}=M \backslash P \rightarrow \mathbf{L}^{3}$, the flux vector of $q$ is defined by

$$
\varphi_{q}:=\operatorname{Im} \oint_{\gamma_{q}} \alpha,
$$

where $\gamma_{q}$ is a positively-oriented curve surrounding $q$.

PROPOSITION 3.2. For the flux vector $\varphi_{q}$ of the simple end $q \in P$ of a maximal map $f$, if $f$ is asymptotic to $(I)(c \neq 0)$ in Theorem 2.7 on a neighborhood of $q$, then

$$
\varphi_{q}=\oint_{\gamma_{q}} \vec{n} d s
$$

where $\vec{n}$ is the conormal vector along $\gamma_{q}$ that is $\left(\gamma_{q}^{\prime}, \vec{n}\right)$ is positively oriented.

Proof. Rewrite $\varphi:=\varphi_{q}$ and let $z(t)$ be a parameterization of $\gamma_{q}$. Put $z(t):=$ $u(t)+\sqrt{-1} v(t)$ and define a positively-oriented orthonormal basis $\left\{\boldsymbol{e}_{1}, \boldsymbol{e}_{2}, v\right\}$ by

$$
\boldsymbol{e}_{1}:=e^{-\lambda} \frac{\partial f}{\partial u}, \quad \boldsymbol{e}_{2}:=e^{-\lambda} \frac{\partial f}{\partial v}
$$

$\nu$ : the limit normal vector at $q$, where $d s^{2}=e^{2 \lambda} d z d \bar{z}$. 
Then

$$
\begin{aligned}
\frac{d f}{d t}= & \frac{\partial f}{\partial z} \frac{d z}{d t}+\frac{\partial f}{\partial \bar{z}} \frac{d \bar{z}}{d t} \\
= & \frac{1}{2}\left(\frac{\partial f}{\partial u}-\sqrt{-1} \frac{\partial f}{\partial v}\right)\left(\frac{d u}{d t}+\sqrt{-1} \frac{d v}{d t}\right) \\
& +\frac{1}{2}\left(\frac{\partial f}{\partial u}+\sqrt{-1} \frac{\partial f}{\partial v}\right)\left(\frac{d u}{d t}-\sqrt{-1} \frac{d v}{d t}\right) \\
= & \frac{\partial f}{\partial u} \frac{d u}{d t}+\frac{\partial f}{\partial v} \frac{d v}{d t} \\
= & e^{\lambda}\left(\boldsymbol{e}_{1} \frac{d u}{d t}+\boldsymbol{e}_{2} \frac{d v}{d t}\right) .
\end{aligned}
$$

Hence,

$$
\frac{d f}{d t} \times v=e^{\lambda}\left(-\boldsymbol{e}_{2} \frac{d u}{d t}+\boldsymbol{e}_{1} \frac{d v}{d t}\right)
$$

and

$$
\left|\frac{d f}{d t}\right|=e^{\lambda} \sqrt{\left(\frac{d u}{d t}\right)^{2}+\left(\frac{d v}{d t}\right)^{2}}=e^{\lambda}\left|\frac{d z}{d t}\right| .
$$

Therefore,

$$
\begin{aligned}
\vec{n} & =\left|\frac{d f}{d t}\right|^{-1} \frac{d f}{d t} \times v \\
& =\left|\frac{d z}{d t}\right|^{-1}\left(\boldsymbol{e}_{1} \frac{d v}{d t}-\boldsymbol{e}_{2} \frac{d u}{d t}\right)
\end{aligned}
$$

Moreover,

Therefore,

$$
d s=\left|\frac{d f}{d t}\right| d t=e^{\lambda}\left|\frac{d z}{d t}\right| d t .
$$

$$
\begin{aligned}
\vec{n} d s & =e^{\lambda}\left(\boldsymbol{e}_{1} d v-\boldsymbol{e}_{2} d u\right) \\
& =\frac{\partial f}{\partial u} d v-\frac{\partial f}{\partial v} d u \\
& =\frac{-\sqrt{-1}}{2}\left(\frac{\partial f}{\partial z}+\frac{\partial f}{\partial \bar{z}}\right)(d z-d \bar{z})-\frac{\sqrt{-1}}{2}\left(\frac{\partial f}{\partial z}-\frac{\partial f}{\partial \bar{z}}\right)(d z+d \bar{z}) \\
& =-\sqrt{-1}\left(\frac{\partial f}{\partial z} d z-\frac{\partial f}{\partial \bar{z}} d \bar{z}\right) \\
& =\operatorname{Im} \frac{\partial f}{\partial z} d z .
\end{aligned}
$$


By definition, the flux vector of $q$ is written as the residue of $\alpha$ at $z=q$ :

$$
\varphi_{q}=2 \pi \operatorname{Res}(\alpha(z) ; q) \text {. }
$$

By the property of the residue, if $M$ is compact, then $\sum_{q \in P} \varphi_{q}=\mathbf{0}$ (balancing formula).

Furthermore, in the proof of Theorem 2.7, $\alpha$ is written as

$$
\alpha=\left(\frac{1}{(z-q)^{2}}(\boldsymbol{a}+\sqrt{-1} \boldsymbol{b})+\frac{1}{z-q} \boldsymbol{c}+\cdots\right) d z .
$$

Therefore,

$$
\varphi_{q}=2 \pi c .
$$

Hence we can rewrite the three types of Theorem 2.7 as in the following theorem. (See Table 1.)

Theorem 3.3. Let $\Delta:=\{z \in \mathbb{C} ;|z|<1\}$ and $\Delta^{*}:=\Delta \backslash\{0\}$. Assume $f: \Delta^{*} \rightarrow \mathbf{L}^{3}$ is a maximal map such that the end 0 is simple. Then $f$ is asymptotic to (I) (respectively (II), (III)) $(c \neq 0)$ of Theorem 2.7 if and only if the flux vector $\varphi_{0}$ is time-like (respectively space-like, null).

Acknowledgement. The author thanks Professor Shin Kato for valuable suggestions and encouragement.

\section{REFERENCES}

[1] T.Imaizumi. Maximal surfaces with conelike singularities of finite type. Kobe J. Math. 18 (2001), 51-60.

[2] S. Kato. Construction of $n$-end catenoids with prescribed flux. Kodai Math. J. 18 (1995), 86-98.

[3] S. Kato, M. Umehara and K. Yamada. An Inverse problem of the flux for minimal surfaces. Indiana U. Math. J. 46 (1998), 529-559.

[4] O. Kobayashi. Maximal surfaces in the 3-dimensional Minkowski space $L^{3}$. Tokyo J. Math. 6 (1983), 297-309.

[5] O. Kobayashi. Maximal surfaces with conelike singularities. J. Math. Soc. Japan 36 (1984), 609-617.

[6] M. Kokubu, M. Umehara and K. Yamada. Minimal surfaces that attain equality in the ChernOsseaman Inequality. Differential Geometry and Integral Systems (Contemporary Mathematics, 308), Ed. M. Guest, R. Miyaoka and Y. Ohnita. American Mathematical Society, 2002, pp. 223-228.

Taishi Imaizumi

Department of Systems and Information Graduate School of Science and Technology

Kumamoto University

2-39-1 Kurokami, Kumamoto 860-8555 Japan

(E-mail:taizo@math.sci.kumamoto-u.ac.jp) 